\title{
XL. On the correction of observed latitudes
}

\section{Colonel A.R. Clarke C.B. F.R.S.}

To cite this article: Colonel A.R. Clarke C.B. F.R.S. (1877) XL. On the correction of observed latitudes, Philosophical Magazine Series 5, 4:25, 302-305, DOI: $10.1080 / 14786447708639342$

To link to this article: http://dx.doi.org/10.1080/14786447708639342

$$
\text { 曲 Published online: } 13 \text { May } 2009 .
$$

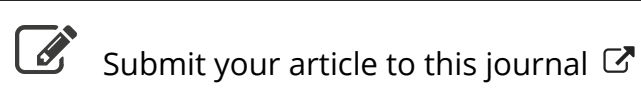

\footnotetext{
Џll Article views: 2
}

Q View related articles $\sqsubset$ 
nished energy would prevent my engaging in any scientific controversy, were any called for.

London, September 17, 1877.

Robert Mallet.

XL. On a Correction to Observed Latitudes.

$B y$ Colonel A. R. Crarke, C.B., F.R.S.

T do not know that any demonstration has ever been given of a theorem enunciated by Gauss in a letter addressed by him in 1853 to General Baeyer. It appears from the letter. in question (of which a translation appeared in the Comptes Rendus des Séances de la Commission permanente de l'Association Géodésique internationale tenues à Florence en 1869) that, at the suggestion of General Baeyer, Gauss had investigated the influence of altitude above the sea-level on observed latitudes, and had arrived at a result which, if not very important to geodesists and astronomers, still is very curious and interesting. The point in question is this :-In the adjoining diagram let $\mathrm{A} \mathrm{PE}$ be a quadrant of a meridional section of the earth, N P P' the normal at any point $P$. Let $\mathrm{P} \mathrm{N}$ represent gravity at $\mathrm{P}$; and on the same scale let $\mathrm{PH}$, parallel to the equator $\mathrm{CE}$, represent the centrifugal force at the same point; then completing the parallelogram $G H, P G$ will be the attraction of the earth. At a point $\mathrm{P}^{\prime}$ vertically above $\mathrm{P}$ the centrifugal force $\mathrm{PH}$ is increased to $\mathrm{P}^{\prime} \mathrm{H}^{\prime}$, retaining its parallelism; and the attraction $P G$ is

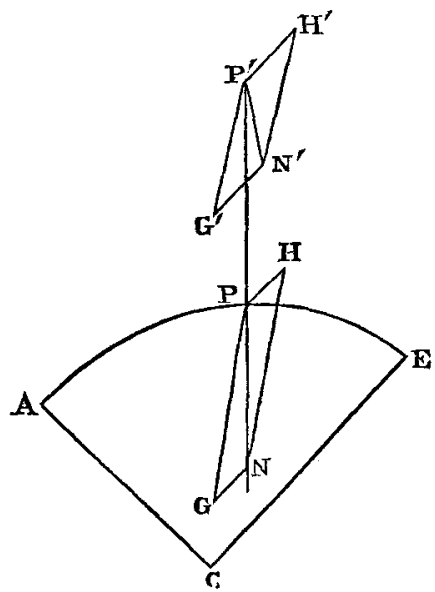

diminished to $\mathrm{P}^{\prime} \mathrm{G}^{\prime}$-not, however, parallel to its former direction, for $G P G^{\prime} P^{\prime}$ converge towards the zenith. The resultant gravity $\mathrm{P}^{\prime} \mathrm{N}^{\prime}$ at $\mathrm{P}^{\prime}$ is therefore no longer of necessity in the line $\mathrm{P}^{\prime} \mathrm{P}$; and the point to be determined is the magnitude of the angle $\mathrm{P} \mathrm{P}^{\prime} \mathrm{N}^{\prime}$.

The potential of the spheroid $\left(x^{2}+y^{2}\right)(1-2 \epsilon)+z^{2}=c^{2}$, whose

* Communicated by the Author. 
semiaxes are $c$ and $c(1+\epsilon)$, $\epsilon$ being a very small quantity whose square may be neglected, at an external point whose coordinates are $f, 0, h$, is

$$
\mathrm{V}=\frac{\mathrm{M}}{r}\left\{1+\frac{\epsilon c^{2}}{5 r^{4}}\left(f^{2}-2 h^{2}\right)\right\},
$$

where $\mathrm{M}$ is the mass of the spheroid ; if $\rho$ be the density,

$$
\mathrm{M}=\frac{4}{3} \pi \rho c^{3}(1+2 \epsilon) \text {. }
$$

If the spheroid, instead of having a uniform density $\rho$, be formed of homogeneous spheroidal shells having a common centre and axis of rotation, the ellipticity as well as the density of these shells varying from the centre to the outer surface, and so being a function of the variable polar semiaxes $c^{\prime}$, then it is easy to see that the potential of such a heterogeneous spheroid is

$$
\mathrm{V}=\frac{4 \pi}{3} \cdot \frac{\phi(c)}{r}+\frac{4 \pi}{15} \psi(c) \frac{f^{2}-2 h^{2}}{r^{5}},
$$

where $c$ is the polar semiaxis of the outer surface, and

$$
\phi(c)=\int_{0}^{c} \rho \frac{d \cdot c^{\prime 3}(1+2 \epsilon)}{d c^{\prime}} d c^{\prime}: \psi(c)=\int_{0}^{c} \rho \frac{d\left(c^{\prime 5} \cdot \epsilon\right)}{d c^{\prime}} d c^{\prime} .
$$

In the theory of the figure of the earth it is shown that the condition of fluid-equilibrium implies the relation

$$
\left(\epsilon-\frac{m}{2}\right) c^{2} \phi(c)-\frac{3}{5} \psi(c)=0,
$$

$\epsilon$ being the ellipticity of the external surface, and $m$ the ratio of the centrifugal force at the equator to the attraction of the earth there. The value of $m$ is, neglecting small quantities of the second order, given by the equation

$$
\frac{4 \pi m}{3} \cdot \frac{\phi(c)}{c^{3}}=\omega^{2},
$$

where $\omega$ is the angular velocity of the earth. Now, since $\mathrm{M}=\frac{4}{3} \pi \phi(c)$, we get for $\mathrm{V}$ the form

$$
\mathrm{V}=\frac{\mathrm{M}}{r}+\frac{\mathrm{M}}{3} c^{2}\left(\epsilon-\frac{m}{2}\right) \frac{f^{2}-2 h^{2}}{r^{5}} \text {. }
$$

This value of the potential is arrived at on the hypothesis of a certain distribution of matter in the interior of the earth; but it is important to remark that it has been obtained by Prof. Stokes (Cambridge and Dublin Mathematical Journal, vol. iv. p. 207) without any such hypothesis as to the distribution of density, be the interior fluid or solid, but assuming only that the surface is a spheroid of equilibrium of small ellipticity. 
Now let $X, Z$ be the components of the attraction of the earth at $P$ parallel respectively to the equator and to the polar axis, $\mathrm{F}$ the centrifugal force at $\mathrm{P}$; then, $\phi$ being the latitude of $\mathrm{P}$, we have the necessary relation $(\mathrm{X}-\mathrm{F}) \tan \phi=\mathrm{Z}$. At $\mathrm{P}^{\prime}$, instead of $\mathrm{X}-\mathrm{F}$ and $\mathrm{Z}$, we have $\mathrm{X}-\mathrm{F}+\delta(\mathrm{X}-\mathrm{F})$ and $\mathrm{Z}+\delta \mathrm{Z}$; and these will correspond, not to $\tan \phi$ but to $\tan (\phi+\delta \phi)$. Hence, differentiating, we have

$$
(\delta \mathrm{X}-\delta \mathrm{F}) \sin \phi-\delta \mathrm{Z} \cos \phi=-\mathrm{Z} \operatorname{cosec} \phi \delta \phi \text {. }
$$

Now $\mathrm{X}=-\frac{d \mathrm{~V}}{d f}$, and $\mathrm{Z}=-\frac{d \mathrm{~V}}{d h}$; and if we put $n$ for the height $\mathrm{P}^{\prime}$, we have in passing from $\mathrm{P}$ to $\mathrm{P}^{\prime}$,

and

$$
\delta f=n \cos \phi, \quad \delta h=n \sin \phi,
$$

whence

$$
\begin{aligned}
& -\delta \mathrm{X}=n \cos \phi \frac{d^{2} V}{d f^{2}}+n \sin \phi \frac{d^{2} \mathrm{~V}}{d f d h}, \\
& -\delta \mathrm{Z}=n \cos \phi \frac{d^{2} V}{d f d h}+n \sin \phi \frac{d^{2} V}{d h^{2}},
\end{aligned}
$$

$\frac{\mathrm{Z}}{n \sin \phi} \delta \phi=\sin \phi \cos \phi\left(\frac{d^{2} \mathrm{~V}}{d f^{2}}-\frac{d^{2} \mathrm{~V}}{d h^{2}}\right)+\frac{d^{2} \mathrm{~V}}{d f d h}\left(\sin ^{2} \phi-\cos ^{2} \phi\right)$ $+\omega^{2} \sin \phi \cos \phi$.

The values of the differential coefficients are found to give

$$
\begin{aligned}
\frac{d^{2} \mathrm{~V}}{d f^{2}}-\frac{d^{2} \mathrm{~V}}{d h^{2}} & =3 \mathrm{M} \frac{f^{2}-h^{2}}{r^{5}}+\mathrm{M} c^{2}\left(\epsilon-\frac{m}{2}\right) \frac{7 f^{4}+12 h^{4}-51 f^{2} h^{2}}{r^{4}}, \\
\frac{d^{2} \mathrm{~V}}{d f d h} & =3 \mathrm{M} \frac{f h}{r^{5}}+\mathrm{M} c^{2}\left(\epsilon-\frac{m}{2}\right) \frac{15 f^{2}-20 h^{2}}{r^{9}} f h,
\end{aligned}
$$

where $r^{2}=f^{2}+h^{2}$. Also

$$
\begin{aligned}
& \sin \phi=\frac{h}{r}\left(1+2 \epsilon \frac{f^{2}}{r^{2}}\right), \\
& \cos \phi=\frac{f}{r}\left(1-2 \epsilon \frac{h^{2}}{r^{2}}\right) .
\end{aligned}
$$

Substituting these in the expression for $\delta \phi$, the result after some little reduction is

$$
\frac{\mathrm{Z}}{\sin \phi} \delta \phi=n \frac{\mathrm{M}}{c^{5}} f h\left(6 \epsilon-8\left(\epsilon-\frac{m}{2}\right)+m\right) .
$$

Now, with sufficient approximation, 


$$
\begin{aligned}
\frac{\mathrm{Z}}{\sin \phi} & =\frac{\mathrm{M}}{c^{2}} ; \\
\therefore \delta \phi & =\frac{n}{c}(5 m-2 \epsilon) \frac{f c}{c^{2}} \\
& =\frac{n}{c}\left(\frac{5}{2} m-\epsilon\right) \sin 2 \phi ;
\end{aligned}
$$

or, if $g^{\prime}$ and $g$ be the values of gravity at the pole and at the equator respectively, then, making use of Clairaut's theorem,

$$
\delta \phi=\frac{n}{c} \cdot \frac{g^{\prime}-g}{g} \sin 2 \phi \text {. }
$$

This result is certainly remarkable for its simplicity; but, practically, for ordinary mountain-heights it only amounts to a small fraction of a second.

\section{Notices respecting New Books.}

An Elementary Treatise on the Dynamics of a System of Rigid Bodies. With Numerous Examples. 'By Edward JoHN Routh, M.A., F.R.S., F.R.A.S., F.G.S., \&c., late Fellow and late Assistant Tutor of St. Peter's College, Canbridge ; late Examiner in the University of London. Third Edition, Revised and Enlarged. London: Macmillan and Co. 1877. (8vo, pp. 564.)

M R. ROUTH'S Treatise on Rigid Dynamics has been for several 1 years the Text Book commonly used by students who "take up" that branch of mechauical science; so that it may be presumed that those who have paid any attention to the subject, are more or less acquainted with one or other of the earlier editions. The present edition, the third, appears in a different form from those which preceded it, and has been very considerably enlarged. In fact, when both the size of the pages and their number are taken into account, we shall not be far wrong in stating that it is larger by one half than the second edition. So that it is a very large volume; and, further, it contains much more matter than the mere number of the pages might lead one to expect.

The work is designed to meet the wants of two classes of students - those who wish for information on the higher parts of Dynamics, and those "to whom the subject is entirely new." The parts to which the attention of the latter class should be directed are indicated by a selection of articles comprising about a third of the volume. The remaining two thirds contain most of the additions. The author's aim in making these additious has been to give the student at least some notion of the most important applications of the general doctrines of Dynamics to particular cases; so that, although such a subject as rigid dynamics might at first sight be thought to be contained within narrow limits, yet in reality the author has had to deal with an extensive literature of

Phil. Mag. S. 5. Vol. 4. No. 25. Oct. 1877. $\mathrm{X}$ 